\title{
Identificação computadorizada de crises tipo ausência no eletrocorticograma de ratos
}

\author{
Automatized identification of absence-like \\ seizures in electrocorticogram of rats
}

\author{
Rodrigo Pavão1, Arnaldo Cheixas-Dias
}

Pavão R, Cheixas-Dias S. Identificação computadorizada de crises tipo ausência no eletrocorticograma de ratos. Rev Med (São Paulo). 2010 jan.-mar.;89(1):12-20.

RESUMO: Crises tipo epilepsia de ausência são expressas no eletrocorticograma de ratos como surtos paroxísticos de descargas espícula-onda. O objetivo do presente trabalho foi desenvolver e testar um sistema automatizado de identificação de crises. O princípio do sistema é distinguir sinais de crise dos demais sinais pela análise da potência na faixa de frequência entre 7 e $11 \mathrm{~Hz}$, que é alta apenas durante as crises. A rotina, elaborada no Matlab $\circledast$, é informada com um intervalo de registro em que não ocorreram crises (crivo), a fim de estabelecer o critério de identificação. O sistema compara, por meio da transformada rápida de Fourier, o crivo com o registro inteiro. A saída do programa é uma tabela informando a presença de crises nesses intervalos. Quatro ratos Wistar geneticamente portadores de crises tipo ausência e implantados com eletrodos corticais tiveram eletrocorticograma por 80 minutos. As crises foram quantificadas manualmente minuto a minuto. O registro e a quantificação das crises de um animal foram usados no refinamento do sistema. Os valores da quantificação automatizada foram bastante similares aos da quantificação manual para o animal usado na elaboração do sistema. Os registros dos demais animais foram usados para testar o sistema e apresentaram padrão similar. O sistema é capaz de quantificar com grande fidelidade as descargas eletrocorticográficas espícula-onda, mostrando-se como potente instrumento na análise de registros em estudos com modelos animais dessa patologia. Adicionalmente, pequenas adaptações permitem identificar outros padrões encontrados em registros eletroencefalográficos (sono, vigília, manifestações patológicas). A rotina está disponível para download em www.ib.usp. br/ rpavao.

DESCRITORES: Eletroencefalografia; Epilepsia tipo ausência/patologia; Padrão de eletrocardiograma/utilização; Disposição de identificação por radiofrequência/utilização Ratos Wistar, Modelos animais.

\footnotetext{
A versão inicial desse trabalho foi apresentado no I Congresso IBRO/LARC de Neurociências da América Latina, Caribe e Península Ibérica (Neurolatam), Búzios, RJ, set. 2008.

1. Laboratório de Neurociências e Comportamento, Departamento de Fisiologia Geral, Instituto de Biociências, Universidade de São Paulo.

Endereço para correspondência: Rodrigo Pavão. Rua Matão, travessa 14, 321, sala 200-B. 05508-900. São Paulo - SP. e-mail: rpavao@gmail.com
} 


\section{INTRODUÇÃO}

$\mathrm{O}$ cérebro exerce controle sobre o organismo por meio da atividade coletiva de populações de células nervosas, que disparam de forma sincrônica em diferentes situações ${ }^{6}$. É possível avaliar essa atividade coletiva por meio do eletroencefalograma (EEG), que é o registro da diferença de potencial elétrico entre dois pontos do sistema nervoso. Assim, o EEG permite a identificação de diferentes ritmos de disparo associados a diferentes níveis de consciência e a estados patológicos, como é o caso da epilepsia, que consiste na sincronização anormal da atividade de populações celulares. Na clínica e na pesquisa básica, a detecção da epilepsia tem no EEG sua principal ferramenta ${ }^{7}$.

Em colônias de diversas linhagens de ratos utilizados em experimentação, há animais portadores de crises tipo ausência, uma patologia de natureza genética caracterizada pela manifestação paroxística de surtos de descargas eletroencefalográficas espícula-onda ${ }^{5,12}$.Tais descargas são consideradas como modelo da epilepsia de ausência e sua incidência pode atingir entre 10 e $20 \%$ dos animais com idade ao redor dos 100 dias $^{4}$. O estudo dos traçados desses animais tem-se mostrado importante para a compreensão dos aspectos fisiopatológicos da epilepsia de ausência.

Embora a análise visual permita a fácil identificação da crise no traçado eletroencefalográfico (Figura 1A), há alguns inconvenientes nesse tipo de estratégia: impossibilidade de avaliar aspectos sutis da patologia (por exemplo, diferença na frequência das oscilações em duas condições diferentes); avaliação discrepante na leitura do traçado por especialistas independentes, já que há critérios subjetivos ${ }^{1}$; e longo tempo investido na leitura ${ }^{7}$.
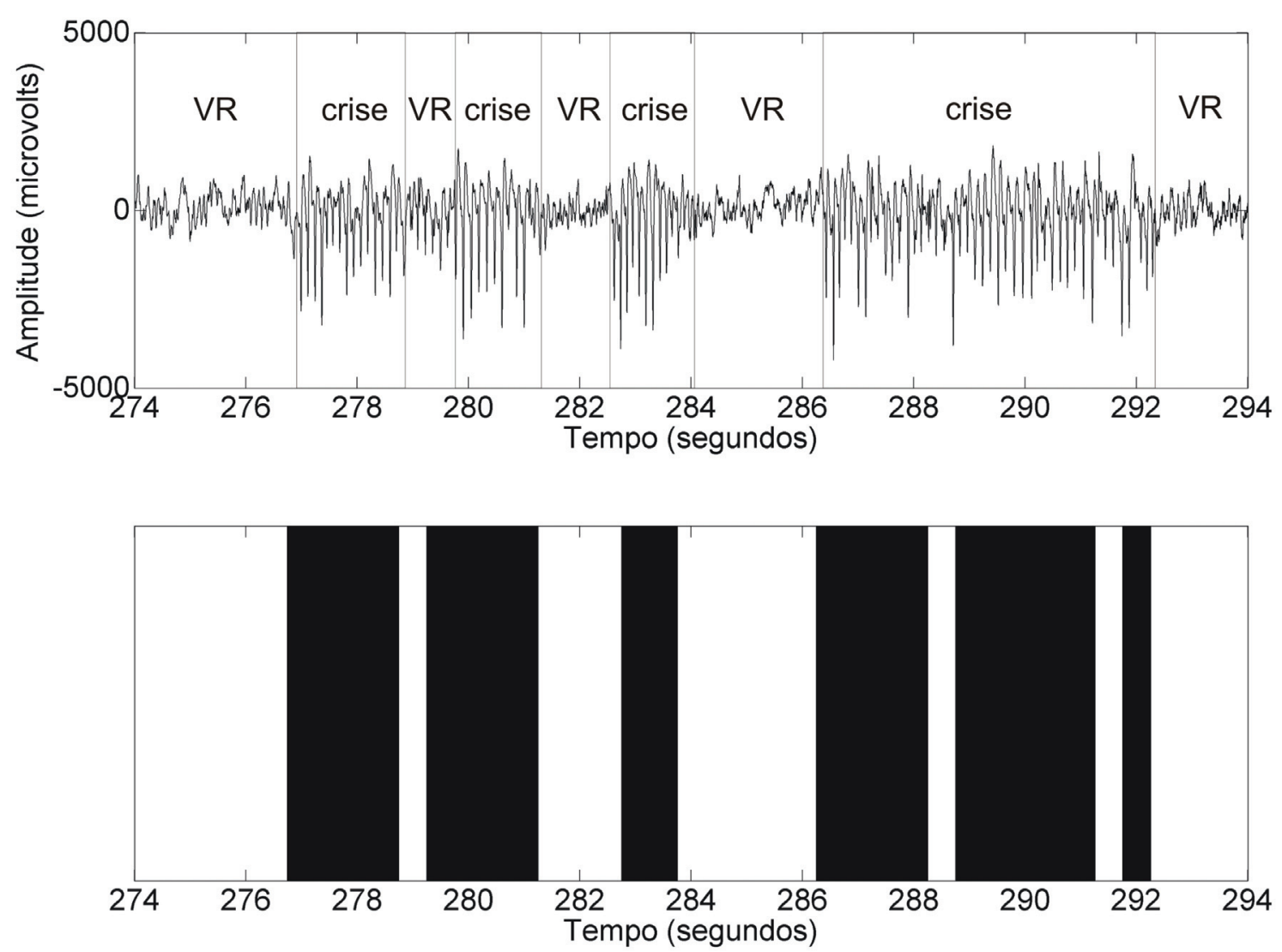

Figura 1. Traçado eletroencefalográfico de rato Wistar porţador de crise tipo ausência com crises discriminadas visualmente da vigília relaxada (VR) (acima). Discriminação automatizada desse traçado dada por rotina apresentada adiante (abaixo) 
Pavão R, Cheixas-Dias A. Identificação computadorizada de crises tipo ausência.

Na análise do traçado eletrofisiológico, há dois aspectos fundamentais: a frequência das oscilações, medida em hertz $(\mathrm{Hz})$, e a amplitude do sinal, medida em volts (V). Esses parâmetros permitem conhecer a identidade do sinal e discriminar diferentes padrões, que são associados a distintos processos fisiológicos e a diferentes fases do ritmo vigília-sono ${ }^{3}$. Existem métodos matemáticos e computacionais para se analisar tais parâmetros, sendo que o mais amplamente utilizado é a transformada rápida de Fourier (FFT), o qual permite identificar os diferentes padrões, convertendo um sinal dentro de séries contínuas de ondas senóides (picos), cada uma delas expressando o "peso" de cada frequência na série estudada ${ }^{2,13}$.

Embora a FFT apresente limitações por ser muito sensível a ruídos de registro e se aplicar mais adequadamente a oscilações estacionárias, ela tem a vantagem de ser fácil e rapidamente aplicável com o uso do computador ${ }^{8}$. Essa característica torna a FFT uma alternativa interessante à análise visual, permitindo que se gaste menos tempo com a quantificação, bem como evitando erros não sistemáticos presentes na análise manual.

Uma crítica frequente do uso da FFT na análise espectral é que sua resolução (capacidade de distinguir dois picos muito próximos) é baixa ${ }^{10}$. Esse problema acontece exclusivamente quando há uma sequência muito curta de dados disponível para análise. Esse é um problema real na avaliação da variação temporal do sinal, uma vez que, mesmo que a série temporal seja longa, ela é subdividida em intervalos curtos para análise.

Essa limitação, no entanto, não torna a FFT incapaz de discriminar padrões mais evidentes; além disso, há variações sutis nessa ferramenta (que foram implementadas no presente estudo - ver métodos) que permitem reduzir esse problema.

Nesse artigo, defendemos a utilização da FFT como ferramenta prática para identificação e contagem de crises de ausência no EEG por meio de uma rotina informatizada de elaboração e manuseio simples, comparando a contagem via FFT com a contagem manual de traçados com crises de ausência (Figura 1).

\section{MÉTODOS}

Quatro ratos Wistar, machos, naturalmente epilépticos de colônia da FMUSP, com 3 meses e cerca de $300 \mathrm{~g}$, foram anestesiados com cetamina $(100 \mathrm{~g} / \mathrm{Kg})$ e tiveram eletrodos de níquel-cromo implantados no córtex frontal. Após recuperação dos animais, que durou 7 dias, o sistema de coleta EMSA BrainNet36 foi conectado aos implantes dos animais, que permaneceram na caixa habitual. A atividade elétrica foi registrada por 80 minutos. Posteriormente, o tempo de crise (em segundos) em janelas de 1 minuto foi quantificado manualmente por especialista. Essa quantificação manual foi o gabarito com o qual se comparou a quantificação automática (apresentada adiante). O sinal registrado e a quantificação manual do animal $A$ foram usados para os testes iniciais, definição do critério e elaboração do programa final. Os sinais observados consistem da diferença de potencial elétrico entre o córtex frontal (área de manifestação proeminente das descargas espícula-onda) e um ponto neutro no crânio. A taxa de amostragem foi de $200 \mathrm{~Hz}$. O sinal foi representado em um espectrograma, que o caracteriza em um plano tempo-frequência, através do código abaixo:

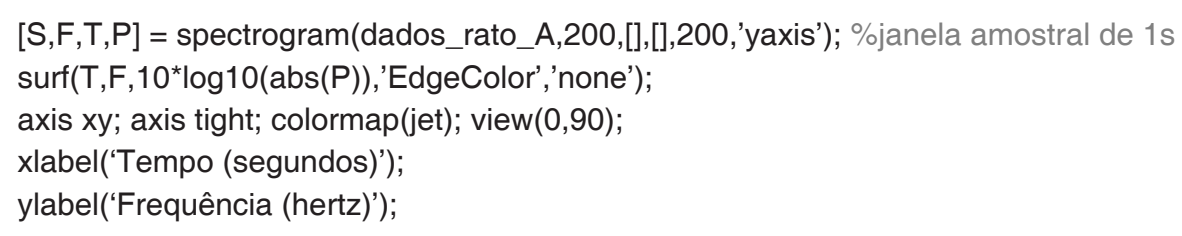

Com o código acima, obtém-se um gráfico como o da Figura 4 (acima). São criadas também as tabelas com as informações base para esse gráfico. Os valores apresentados na tabela e no gráfico consistem na potência do sinal nas frequências obtidas pelo método da short time FFT (STFFT) variação da FFT que diminui a limitação imposta pelo uso de janela temporal curta, como citado previamente nesse trabalho. Esse método envolve a multiplicação de um dado sinal cujo espectro se deseja analisar e a aplicação da FFT no sinal multiplicado. Assim, o espectro do STFFT é dependente da janela temporal definida - não é possível analisar frequências baixas com janelas muito pequenas.

A Figura 2 mostra os valores de potência ao longo das frequências em dois momentos distintos, um em vigília relaxada (não crise) e outro em crise epiléptica. A diferença evidente na observação direta do sinal (Figura $1 \mathrm{~A}$ ) também é clara na representação frequência x potência, na qual se observa um pico de potência nos $7 \mathrm{~Hz}$ que decai até cerca de $10 \mathrm{~Hz}$ e que diferencia os estados, o que está de acordo com os dados da literatura desse tipo de crise. 


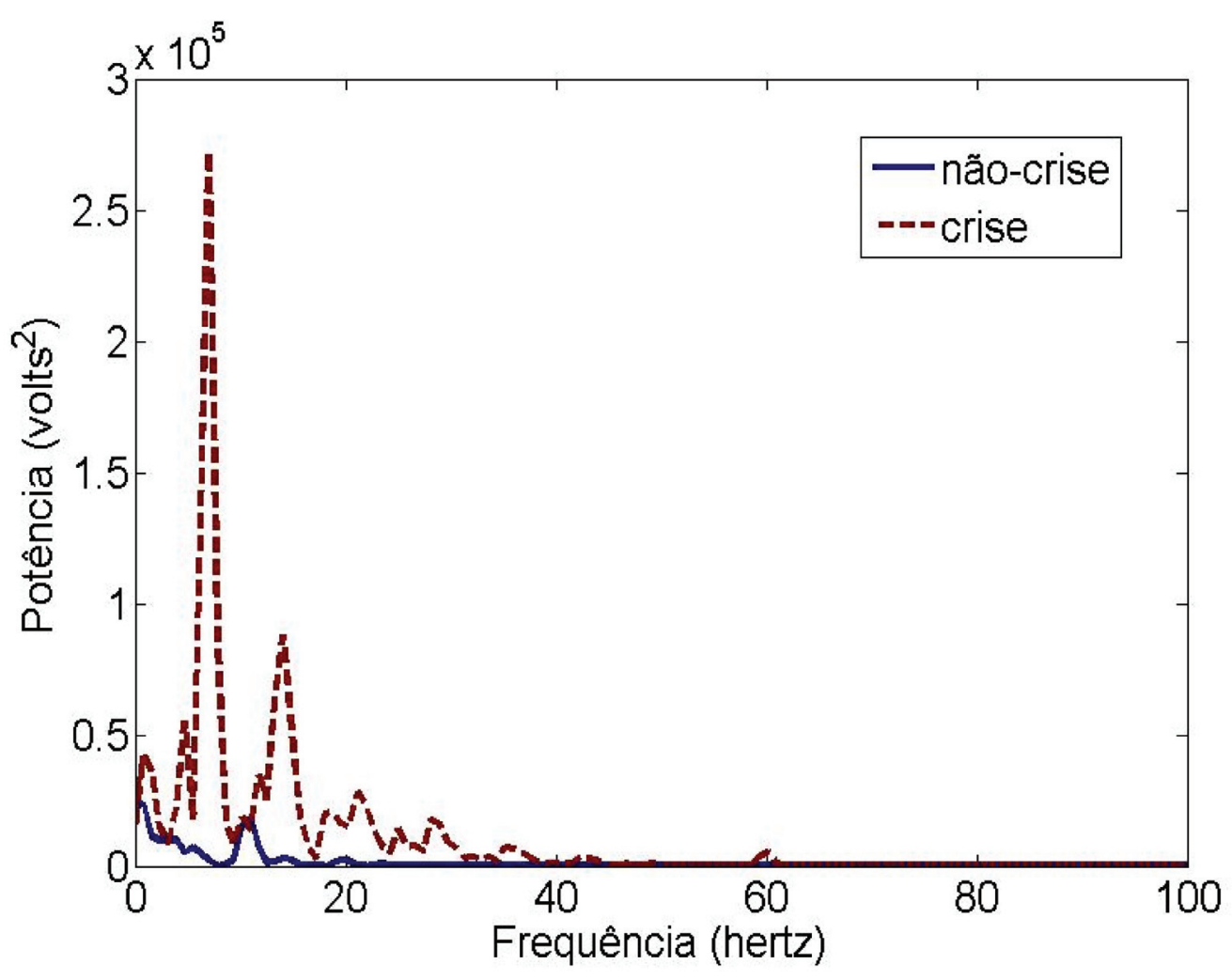

Figura 2. Representação tempo-frequência usando saída da função spectrogram de estados de crise e não-crise do rato $\mathrm{A}$

A estratégia do programa de quantificação automática é usar a diferença de potência nessa frequência para classificar o sinal em crise ou nãocrise; para isso, usa-se a soma das potências nessa faixa de frequências. Um minuto de crise e um minuto de não-crise do rato $A$ foram selecionados para avaliar os valores da soma de potências, que apresentaram uma pequena sobreposição, como mostrado na Figura 3. O critério (dado pela média +2.5 de desvio padrão da amostra não-crise, representado pela linha cinza na figura) foi definindo com o objetivo de minimizar o número de falso-negativos e falso-positivos.

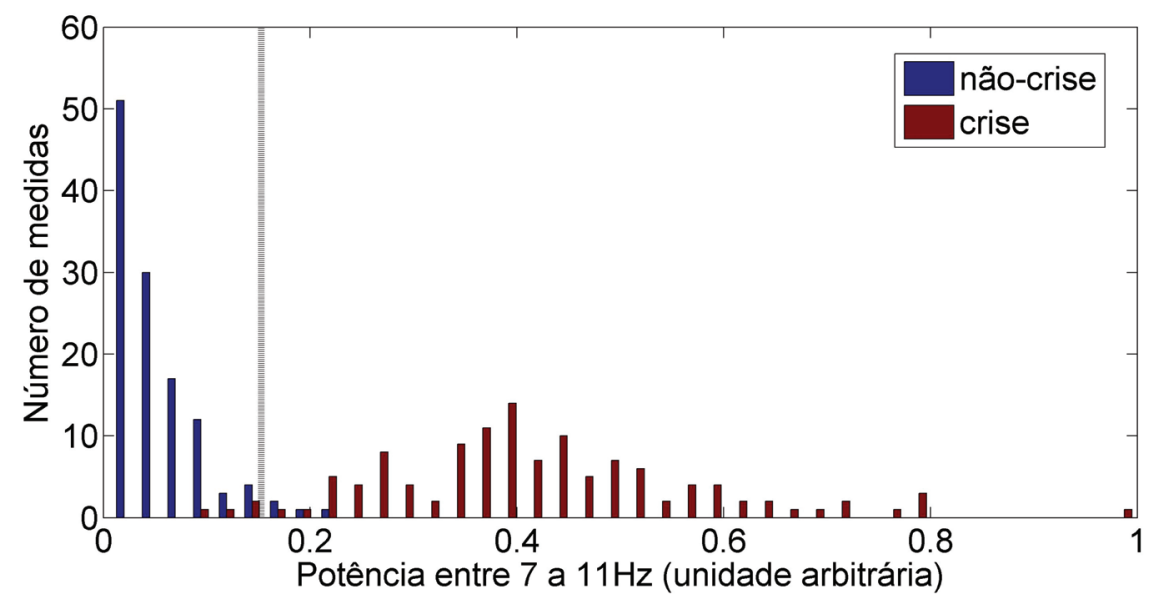

Figura 3. Histograma da potência do sinal na frequência de interesse, analisada para estados de crise e não-crise. O critério ótimo de separação entre os estados, que minimiza o número de falso-positivos e falso-negativos, está representado pela linha vertical cinza 
Pavão R, Cheixas-Dias A. Identificação computadorizada de crises tipo ausência.

Estão definidos os pontos fundamentais do programa: 1) obtenção das potências na faixa de freqüência de 7 a $11 \mathrm{~Hz}$, pela função spectrogram; 2) elaboração do critério, com o qual os valores de potência serão comparados para decidir se o estado do animal naquele instante é de crise ou não-crise. Além disso, o programa tem que apresentar a saída, que é expressa como uma tabela (instante de tempo e classificação em crise ou não-crise) e gráficos (o espectrograma; os valores de potência na frequência de interesse ao longo do tempo e o critério; e uma representação gráfica da tabela em gráfico "código-de-barras"). O programa completo, com os comentários (que são iniciados pelo símbolo “\%”) está apresentado abaixo.

function saída $=$ crise (dados_origem,frequencia_amostragem,sem_crise_inicio,sem_crise_fim)

\% Essa função calcula a soma das amplitudes do sinal entre as frequências 7

$\%$ e $11 \mathrm{~Hz}$ do vetor dado_origem (N linhas x 1).

$\%$ Gera também gráfico com os valores calculados ao longo do tempo.

\% Potências altas nessas frequências são indicativos de crise de ausência.

$\%$

\% O arquivo de saída apresenta em cada linha as seguintes informações:

$\%$ coluna $1=$ momento do registro (segundos)

$\%$ coluna 2 = 0 (não teve crise) ou 1 (teve crise)

$\%$

\% SINTAXE: saída = crise (dados_origem, freq_amostr, sem_crise_inicio, sem_crise_fim)

EM QUE: 'saida' = nome do arquivo de saída

'dados_origem' = nome do arquivo com registro

'freq_amostr' = frequência de amostragem do registro

'sem_crise_inicio' e 'sem_crise_fim' = limites de tempo em que não ocorreram crises (EM SEGUNDOS)

$\%$

criterio_frequencia_min $=10 ; \%$ linha da freq interesse linha $10 \sim 7 \mathrm{~Hz}$ para amostragem de $200 \mathrm{~Hz}$ criterio_frequencia_max $=15 ; \%$ linha da freq interesse linha $15 \sim 11 \mathrm{~Hz}$ para amostragem de $200 \mathrm{~Hz}$

\%calcula a potência em cada frequência ao longo do tempo

[S,F,T,P] = spectrogram (dados_origem, frequencia_amostragem,[],[],frequencia_amostragem,'yaxis');

$\%$ S=espectrograma; $F=$ frequências; $T=$ tempo; $\bar{P}=$ potência

\%grafica a potência em cada frequência ao longo do tempo

figure

subplot $(3,1,1)$

surf (T,F,10*log10(abs(P)),'EdgeColor','none');

axis $x y$; axis tight; colormap(jet); view $(0,90)$;

xlabel ('Tempo (segundos)');

ylabel ('Frequência (hertz)');

saida = T'; \%escreve a coluna 1 do arquivo de saída

P_soma $=$ sum $(P($ criterio_frequencia_min:criterio_frequencia_max,:),1); \%soma das potências na faixa de frequência de interesse

criterio $=$ mean $\left(P \_s o m a\left(r o u n d\left(s e m \_c r i s e \_i n i c i o * 2\right): r o u n d\left(s e m \_c r i s e \_f i m * 2\right)\right)\right)+2.5 * \operatorname{std}\left(P \_s o m a\left(r o u n d\left(s e m \_c r i-\right.\right.\right.$ se_inicio*2):round(sem_crise_fim²))); \%calcula o critério

\%compara P_soma com criterio ao longo do tempo - coluna 2 do arquivo de saída

for $\mathrm{i}=1$ :length(P_soma)

if $\mathrm{P} \_$soma(i) $>$criterio

saida $(\mathrm{i}, 2)=1 ; \% 1=$ teve crise

else

saida $(\mathrm{i}, 2)=0 ; \% 0=$ não teve crise

end

end

\%grafica soma das potências ao longo tempo / critério

P_soma_rankeado = sort $\left(P \_s o m a\right)$; 
$\operatorname{axis1}(1)=0.1 ; \operatorname{axis1}(2)=$ saida $($ length $($ saida $(:, 1)), 1)+1$; axis1 $(3)=0$; axis1 $(4)=P \_s o m a \_r a n k e a d o\left(r o u n d\left(l e n g t h\left(P \_\right.\right.\right.$ soma_rankeado) *0.95));

critplot(1)=criterio; $\operatorname{critplot}(2)=$ criterio;

subplot $(3,1,2)$

plot(axis1(1:2),critplot(1:2),'-r','Linewidth',4)

hold on

plot(T,P_soma,'k.','MarkerSize',4);

axis(axis1);

xlabel('Tempo (segundos)');

set(gca,'YTick','remove')

ylabel('Potência entre 7 e 11Hz');

\%grafica código-de-barras

$\operatorname{axis} 2(1)=0.1 ; \operatorname{axis} 2(2)=$ saida $($ length $(\operatorname{saida}(:, 1)), 1)+1 ; \operatorname{axis} 2(3)=0 ; \operatorname{axis} 2(4)=1$;

subplot $(3,1,3)$

bar(saida(:,1), saida(:,2),1,'k');

axis(axis2);

xlabel('Tempo (segundos)');

set(gca,'YTick','remove')

Os gráficos obtidos rodando essa função com os dados do rato $A$, através do comando
saida=crise(dados_rato_A,200,3500,3700); estão representados na Figura 4.

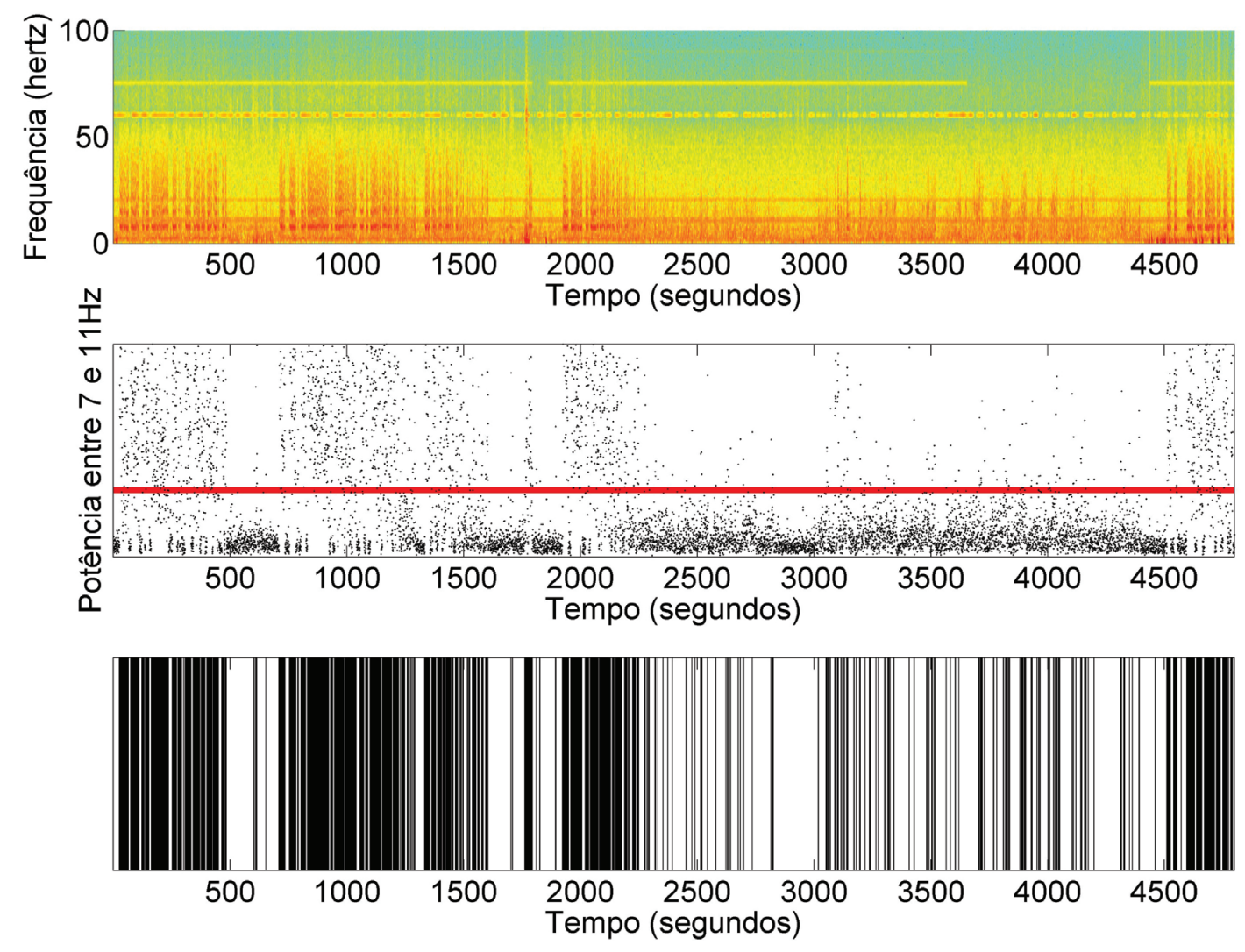

Figura 4. Espectrograma (acima), potência na frequência de interesse ao longo do tempo e o critério (meio), gráfico "código-de-barras" (abaixo) 
Pavão R, Cheixas-Dias A. Identificação computadorizada de crises tipo ausência.

\section{RESULTADOS}

Após o estabelecimento da rotina computacional, foi avaliado se a ferramenta de leitura automática de traçados é capaz de quantificar adequadamente as crises de ausência. Além do rato $A$, que foi usado para avaliações iniciais e para definição do critério, avaliou-se os registros dos ratos $B, C$ e $D$.
Foram comparados os tempos de crise em janelas de 1 minuto ao longo do registro de 80 minutos quantificados automaticamente e manualmente. Essa comparação está representada na Figura 5, para os quatro animais.
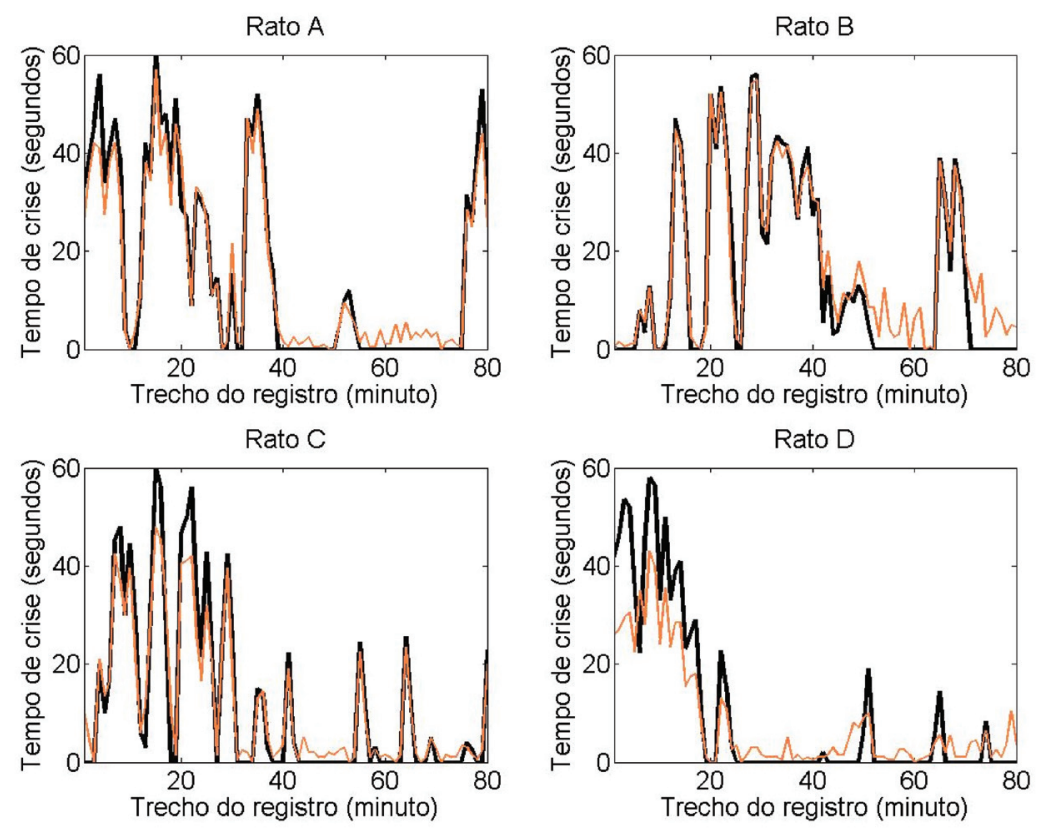

Figura 5. A linha preta, mais grossa, corresponde à quantificação manual das crises; a linha clara, mais fina, corresponde à quantificação automática.

Visualmente, é possível perceber a grande semelhança entre as quantificações manual e automática nos quatro animais. Para quantificar essa semelhança, pode-se avaliar o coeficiente de correlação $\left(R^{2}\right)$ e equação da reta (com quantificação manual no eixo X e automática no eixo Y). Quanto mais próximo de 1 o coeficiente de correlação, de 1 o coeficiente angular e de 0 o intercepto, mais fiel é a quantificação automática (Tabela 1). Esses indicadores confirmam a grande semelhança entre as quantificações manual e automática, o que revela que o sistema é capaz de quantificar com grande fidelidade as descargas eletrocorticográficas espícula-onda, mostrando-se como potente instrumento na análise de registros em estudos com sistemas modelos em animais desta patologia.

Tabela 1. Coeficiente de correlação e equação da reta para quantificações manual e automática nos quatro animais

\begin{tabular}{ccc}
\hline & Coeficiente de correlação (R2) & Equação da reta: $\mathrm{y}=$ coeficiente_angular * $\mathrm{x}+$ intercepto \\
\hline Rato A & 0.9755 & $\mathrm{y}=0.8616 \mathrm{x}+1.7608$ \\
Rato B & 0.9532 & $\mathrm{y}=0.8832 \mathrm{x}+3.7948$ \\
Rato C & 0.9634 & $\mathrm{y}=0.7958 \mathrm{x}+2.0865$ \\
Rato D & 0.9187 & $\mathrm{y}=0.6200 \mathrm{x}+2.0390$
\end{tabular}




\section{DISCUSSÃO}

$\mathrm{Na}$ análise do sinal eletrofisiológico de ratos que expressam crises tipo ausência, identificaramse e quantificaram-se essas crises por meio de um sistema computadorizado que classifica o período como crise ou não-crise a partir da potência do sinal entre 7 e $11 \mathrm{~Hz}$. As crises de ausência apresentam potência alta nessa faixa de frequência. $O$ método usado é de implementação bastante fácil, como pode ser visto no programa para Matlabß apresentado na sessão Métodos. No entanto, esse método apresenta limitações na detecção de sinais menos claros; essas limitações, no entanto, não parecem distorcer as quantificações das crises como os dados demonstram (Figura 5). A quantificação manual das crises, que é procedimento trabalhoso e lento, foi feita por um por um especialista e comparada com a quantificação feita pelo sistema computadorizado, gerando resultados extremamente semelhantes, o que indica que esse método é bastante confiável.

O presente trabalho tem a função de disseminar o uso de métodos computacionais na análise de crises epilépticas. Uma crítica que poderia ser apresentada é que a análise manual é possível e mais confiável do que a análise pela ferramenta aqui apresentada. De fato, a análise manual é possível, mas a confiabilidade desta pode não ser efetiva, uma vez que há variabilidade nas análises feitas por um indivíduo; isso sem considerar que a análise manual é extremamente custosa. Outra crítica comum ao uso da análise espectral do sinal eletrofisiológico de campo para detecção de crises por meio da transformada de Fourier é que há estratégias com maior precisão já desenvolvidas ${ }^{11}$. Há dois fatores, no entanto, que devem ser considerados com respeito a essa questão. Primeiro não se pode desprezar o fato de que embora a precisão do método de Fourier não seja absoluta, ela é significativa $(98 \%)^{7,11}$, de modo que não ocorrem erros de análise de grande magnitude. Esse aspecto é corroborado pelos coeficientes de correlação obtidos nesse trabalho entre as análises visual e via Fourier (entre 0,91 e 0,98). De fato, esse método tem sido usado em trabalhos recentes de pesquisa básica ${ }^{9}$. Outro fator essencial a respeito das possíveis limitações da análise espectral computadorizada por meio da transformada de Fourier é que, embora haja métodos com precisão absoluta já disponíveis, sua aplicação exige um custo maior por parte do usuário, principalmente no que diz respeito aos conhecimentos pré-requeridos em métodos computacionais. Assim, embora o uso da transformada de Fourier a partir de uma rotina em Matlab® implique uma precisão menor que outros métodos, a sua implementação é extremamente simples e acessível ao pesquisador de epilepsia que não tenha muitos conhecimentos em programação. Desse modo, uma grande vantagem da metodologia proposta nesse trabalho é permitir o acesso de muitos pesquisadores de epilepsia a métodos computadorizados quantitativos e qualitativos de epilepsia com autonomia, sem comprometer a fidedignidade das análises realizadas.

Pavão R, Cheixas-Dias S. Automatized identification of absence-like seizures in electrocorticogram of rats. Rev Med (São Paulo). 2010 jan.-mar.;89(1):12-20.

ABSTRACT: Absence-like seizures are presented in rat electrocorticogram as paroxistics spike-wave discharges. The aim of this study was to develop and test an automatized system for seizures identification. System fundament is to distinguish seizure signals from others by power analysis in frequency range between 7 and $11 \mathrm{~Hz}$, which is high only during seizures. Routine, elaborated at Matlab®, is inputted with an interval without seizures (sample) in order to set up the criterion of identification. The system compares, through the Fourier fast transformed, the sample with the entire recording. Program output is a table that reports the presence of seizures in such intervals. Four Wistar rats genetically prone to show absence-like seizures with implanted cortical electrodes were submitted to 80 minutes of electrocorticogram. Seizures were counted manually minute by minute. Recording and seizure quantification of one animal were used to refine the system. Automatized quantification was very similar to manual quantification for the animal used as sample. Recordings of the other animals, used to test the system, shows similar pattern. The system is able to quantify with high fidelity the spike-wave discharges, so that it is a powerful instrument to analyze electroencephalogram in studies with animal models of this pathology. Additionally, minor changes allow identifying other patterns found in electrophysiological recordings (sleep, wakefulness, pathological manifestations etc). The routine is available for download at www.ib.usp.br/ rpavao.

KEY WORDS: Electroencephalography; Epilepsy, absence/pathology; Electrocardiogram pattern/utilization; Radiofrequency identification device/utilization; Rats, Wistar; Models, animal. 
Pavão R, Cheixas-Dias A. Identificação computadorizada de crises tipo ausência.

\section{REFERERÊNCIAS}

1. Alkan A, Koklukaya E, Subasi A. Automatic seizure detection in EEG using logistic regression and artificial neural network. J Neurosci Methods. 2005;148(2):167-76.

2. Cooley JW, John W. Tukey, An algorithm for the machine calculation of complex Fourier series. Math Comput. 1965;19:297-301.

3. Guyton AC, Hall JE. Tratado de fisiologia médica. 11a ed. Rio de Janeiro: Elsevier; 2006.

4. Lenneberg-Hoshino C. Ciclos do sono e envelhecimento em ratos portadores de epilepsia de pequeno mal de ausência [Tese]. Botucatu: Universidade Estadual Paulista; 1999.

5. Marescaux C, Vergnes M, Micheletti G, Depaulis $A$, Reis J, Rumbach $L$, et al. Une forme genetique d'absence petit malchez le rat Wistar. Rev Neurol (Paris). 1984;140:63-6.

6. Martin JH. The collective electrical behavior of cortical neurons: the electroencephalogram and the mechanisms of epilepsy. In: Kandel ER, Schwartz JH, Jessell TM, editors. Principles of neural science. 3rd ed. New York: Elsevier; 1991. p.777-91.

7. Mohseni HR, Maghsoudi A, Shamsollahi MB. Seizure detection in EEG signals: a comparison of different approaches. In: 28th Annual International Conference - IEEE Engeneering in Medicine and Biology Society (EMBS), New York, 30 Aug 3 Sept., 2006. New York,
NY; 2006.

8. Muthuswamy J, Thakor NV. Spectral analysis methods for neurological signals. J Neurosci Methods. 1998;83(1):1-14.

9. Ribeiro S. 2007. EEG em experimentação animal: sono e aprendizado - dinâmica cerebral, conectividade e cognição. In: XXII Reunião Anual da Federação de Sociedades de Biologia Experimental (FESBE). Águas de Lindóia, SP, Brasil, 22-25 ago. 2007.

10. Sinha RK, Ray AK, Agrawal NK. An artificial neural network to detect EEG seizures. Neurol India. 2004;52(3):399-400.

11. Tzallas AT, Tsipouras MG, Fotiadis DI. The use of timefrequency distributions for epileptic seizure detection in EEG recordings. In: Proceedings of the 29th Annual International Conference of the IEEE EMBS - Cité Internationale, Lyon, France, Aug. 23-26, 2007.

12. Vergnes M, Marescaux C, Micheletti G, Reis J, Depaulis $A$, Rumbach $L$, et al. Spontaneous paroxysmal electroclinical patterns in rat: a model of generalized non-convulsive epilepsy. Neurosci Lett. 1982;33(1):97101.

13. Weleratna S. Thirty years of FFT analyzers. Sound and Vibration. 1997 Jan.:1-5. Available from: http:// www.ingelec.uns.edu.ar/pds2803/Materiales/ Historicos/9701wel1.pdf 